\title{
Type II Abernethy malformation in an adult male patient-a rare and reversible cause of pulmonary hypertension: a case report with review of literature
}

\author{
Arjun Agarwal ${ }^{*}$, Durvesh Bhangale, Vinayak M. Sawardekar and Srikar Puvvada
}

\begin{abstract}
Background: Co-occurrence of Type II Abernethy malformation (AM) with Type 1 pulmonary hypertension (PH) is extremely rare. In these patients, management of AM leads to reversal of $\mathrm{PH}$.

Case presentation: We report a case of 24-year-old male that presented with fever and dyspnea of insidious-onset. Initial X-ray chest revealed pulmonary edema, prominent pulmonary knuckle, and dilated right descending pulmonary artery. Two-dimensional echocardiography suggested raised pulmonary artery systolic pressure (145 $\mathrm{mmHg}$ ) and pulmonary angiogram revealed findings suggestive of Type $1 \mathrm{PH}$. Treatment with Tab. TadalafilBosentan (20/5 mg, thrice daily) was initiated, but no major relief was obtained. On day 10, dyspnea worsened. Routine ultrasound revealed splenomegaly. Further evaluation with Doppler of the portal vein was suggestive of portal hypertension, and arterial ammonia was found to be raised. Contrast-enhanced computed tomography of abdomen and pelvis demonstrated a single dilated $(1.3 \mathrm{~cm})$ and torturous venous shunt between right internal iliac vein and superior mesenteric vein. Thus, a definitive diagnosis of Type II AM was reached. The patient was stabilized and subjected to the shunt closure with ASD Cocoon stent graft and recovered well.
\end{abstract}

Conclusion: Our case demonstrates a rare but reversible cause of PH. Type II AM should be included in the differential diagnosis of a patient presenting with dyspnea and provisionally diagnosed as a case of $\mathrm{PH}$.

Keywords: Abernethy malformation, Case report, Congenital, Portal vein, Pulmonary hypertension, Vascular anomaly

\section{Background}

Abernethy malformation (AM), a rare vascular anomaly, allows a direct flow of portal blood into the inferior vena cava (IVC) [1]. Its prevalence is estimated to be 1 per 30,000 live births [2]. Described initially by John Abernethy [3], its two variants, on the basis of porto-caval shunt, were subsequently illustrated by Morgan and Superina [4]. While Type I AM has an end-to-side shunt and absence of intrahepatic portal vein (PV), Type II AM has a side-to-side shunt and presence of hypoplastic intrahepatic PV. It has

\footnotetext{
* Correspondence: arjunagarwal4jun@gmail.com

Department of Medicine, The Grant Government Medical College and Sir J.J. Group of Hospitals, JJ Marg, Nagpada-Mumbai Central, Near Sandhurst Road and J J Police Station, Mumbai 400008, India
}

been reported to result in severe complications including pulmonary hypertension (PH), hepatic encephalopathy (HE), hepatopulmonary syndrome, or hepatic carcinoma. The treatment depends on the variant diagnosed, i.e., from liver transplantation (LT) for Type I to embolization, ligation, or transcatheter closure of shunt for Type II [5]. In this report, we present the diagnostic approach and treatment of an adult male with Type II AM that presented as a case of Type $1 \mathrm{PH}$.

\section{Case presentation}

A 24-year-old male presented with fever and dyspnea for the last 1 month. Fever was low-grade, not associated with chills and rigors, and relieved on taking antipyretics. 
Dyspnea was insidious in onset, gradually progressive which aggravated on exertion and on lying-down, and relieved on taking rest and assuming standing position. There was a history of cough with blood-streaked scanty expectoration for the last 3 days. There was no significant past history. Examination revealed tachycardia and tachypnea, pulse was $110 / \mathrm{min}$, blood pressure $(120 / 70 \mathrm{mmHg})$ and oxygen saturation ( $99 \%$ on room air) were normal. There was no pallor, icterus, cyanosis, clubbing, pedal edema, and generalized lymphadenopathy. On auscultation, normal vesicular breath sounds were audible. There was a wide parasternal heave, with apex beat in the 5th intercostal space along the midclavicular line, and a wide split S2 with loud P2 component. Examination of other systems was within normal limits.

The routine laboratory investigations were normal. The initial X-ray chest (PA view) suggested pulmonary edema, prominent pulmonary knuckle, and dilated right descending pulmonary artery. Electrocardiogram was suggestive of right axis deviation and right ventricular hypertrophy. Two-dimensional echocardiography revealed dilated right atrium and ventricle with dilated pulmonary trunk and branches with 50\% ejection fraction. The pulmonary artery systolic pressure (PASP) was 145 $\mathrm{mmHg}$. Computed tomography pulmonary angiogram (CTPA) demonstrated dilated pulmonary trunk $(38 \mathrm{~mm})$, and right $(32 \mathrm{~mm})$ and left $(33 \mathrm{~mm})$ main pulmonary arteries with patchy areas of ground glass opacities in perihilar region of both right and left lower lobes (Fig. 1). There was no sign suggestive of pulmonary thromboembolism. Thus, the diagnosis of Type $1 \mathrm{PH}$ was reached with the etiology still under evaluation. Antinuclear antibody (ANA), c-anti-neutrophil cytoplasmic antibody (c-ANCA), and p-ANCA were negative and thus, autoimmune causes were ruled out. The patient was initiated on a combination of Tab. Tadalafil-Bosentan (20/5 mg, thrice daily).
On day 10, dyspnea worsened and there was the presence of hemoptysis $(100 \mathrm{ml})$, hypotension $(70 / 50$ $\mathrm{mmHg}$ ), and raised central venous pressure (CVP, >15 $\left.\mathrm{cmH}_{2} \mathrm{O}\right)$. Tab. Tadalafil-Bosentan were stopped due to hypotension. Contrast-enhanced computed tomography (CECT) of the chest demonstrated pulmonary edema, multiple centrilobular nodules with surrounding ground glass opacity, and inter- and intra-lobular septal thickening throughout bilateral lung fields suggesting lower respiratory tract infection. Right atrium and ventricles were dilated. Moreover, dilated pulmonary trunk (38 $\mathrm{mm})$, and right $(31 \mathrm{~mm})$ and left $(29 \mathrm{~mm})$ main pulmonary arteries were suggestive of $\mathrm{PH}$. Thus, the patient was shifted to the intensive care unit and managed conservatively, inotropes (Noradrenaline $1 \mu \mathrm{g} / \mathrm{kg} / \mathrm{min}$ and Dobutamine $5 \mu \mathrm{g} / \mathrm{kg} / \mathrm{min}$ for 2 days, and Noradrenaline $1 \mu \mathrm{g} / \mathrm{kg} / \mathrm{min}$ for next 2 days) were tapered gradually, and pulmonary edema resolved and dyspnea improved, though not significantly. To identify the cause of treatment-resistant Type $1 \mathrm{PH}$, a routine ultrasound of the abdomen was performed that led to an incidental finding of mild splenomegaly $(14.5 \mathrm{~cm})$. Further evaluation was done with Doppler of portal vein and it demonstrated multiple collaterals with mild splenomegaly suggestive of portal hypertension (PHT). Presence of PHT and lung field changes prompted us to perform arterial ammonia and routine hematological investigations. We observed that arterial ammonia $(92 \mu \mathrm{mol} / \mathrm{L})$ and total leucocyte count $\left(17,000 / \mathrm{mm}^{3}\right)$ were raised. CECT of abdomen and pelvis demonstrated features of PHT in the form of mild splenomegaly, and single dilated $(1.3 \mathrm{~cm})$ and torturous venous shunt between the right internal iliac vein and superior mesenteric vein. Moreover, multiple collaterals were seen in the mesorectal fascia. Main portal vein $(11 \mathrm{~mm})$ and its right $(7.5 \mathrm{~mm})$ and left $(7.3 \mathrm{~mm})$ branches were narrow. Thus, a definitive diagnosis of Type II AM was reached (Fig. 2A).
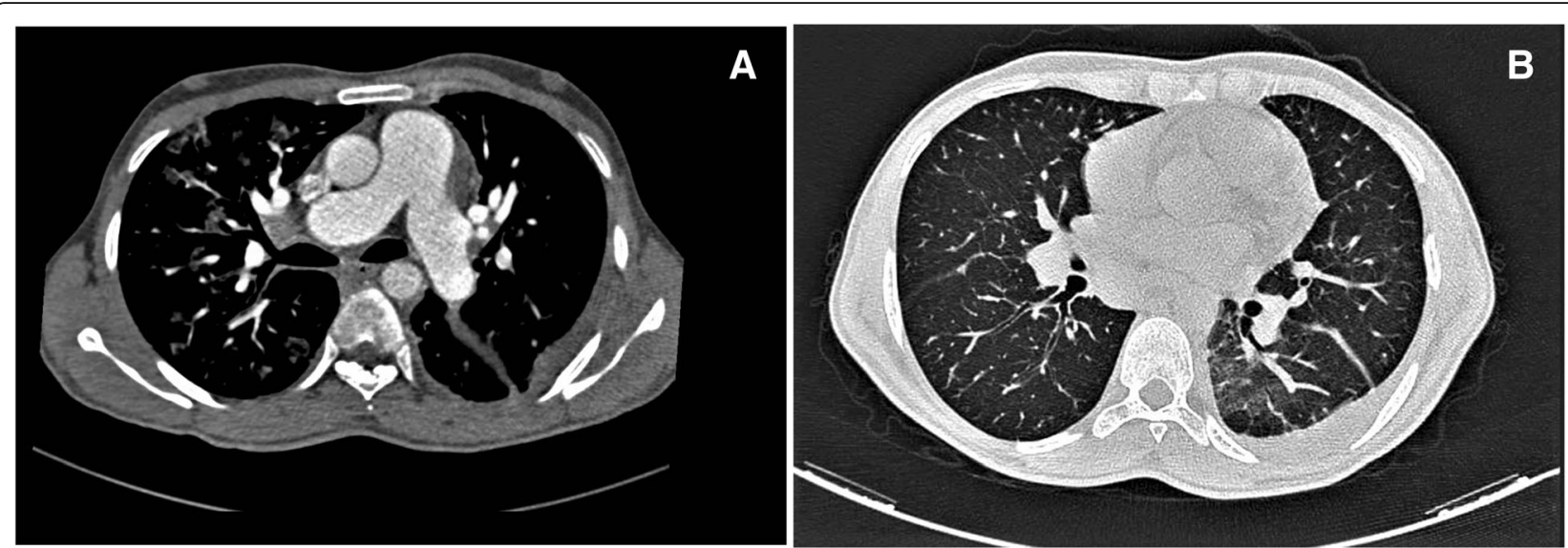

Fig. 1 Computed tomography pulmonary angiogram (CTPA). A Dilated pulmonary trunk (32.2 mm), and right (30.2 mm) and left (30.5 mm) main pulmonary arteries. B Patchy areas of ground glass opacities in the perihilar region of both right and left lower lobe 


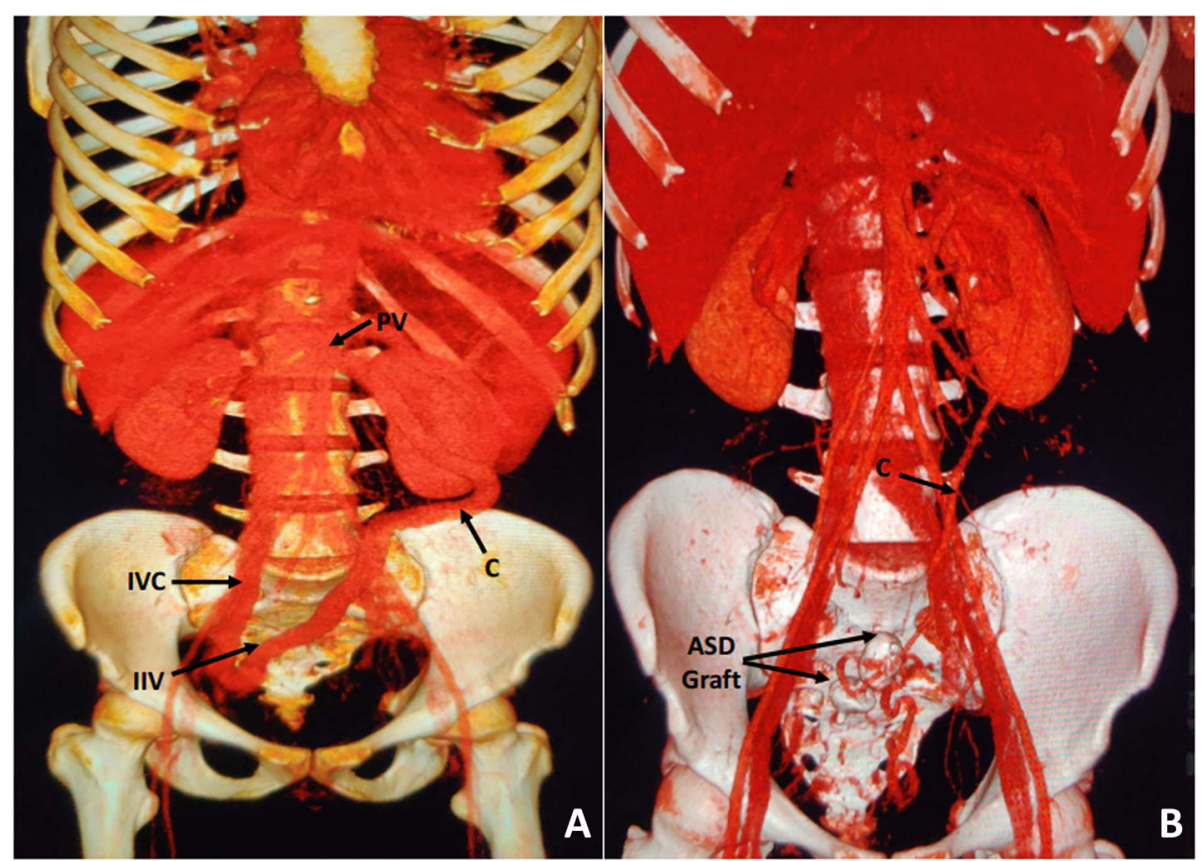

Fig. 2 3D reconstruction CT images illustrating the shunt before and after closure. A Before closure, the shunt was dilated and tortuous (C, venous collateral between right internal iliac vein and superior mesenteric vein; PV, portal vein; IVC, inferior vena cava; and IIV, internal iliac vein). B After closure, the shunt had shrunken significantly (shunt closure with ASD Cocoon stent graft, and C - shrunken collateral)

The patient was stabilized and subjected to the shunt closure with a $32 \times 18 \times 28 \mathrm{~mm}$ ASD Cocoon stent graft (Vascular Concepts Ltd., India), accessed through the right femoral vein (Fig. 2B), while the right internal jugular vein access was taken for monitoring the pressure gradient. The patient tolerated the procedure well and improved symptomatically.

On day 3, post-procedure two-dimensional echocardiography was suggestive of IVC collapsing and PASP of $16 \mathrm{mmHg}$ with no $\mathrm{PH}$. However, the other baselines changes on two-dimensional echocardiography, i.e., dilated right atrium and ventricle with dilated pulmonary trunk and branches, remained the same. On day 9, CECT abdomen and pelvis suggested occlusion of the shunt and demonstrated non-contrast opacification of the shunt distal to the graft. At 3 months follow-up, the patient had no fresh complaints and PASP was normal on two-dimensional echocardiography.

\section{Discussion}

AM occurs due to deviation from the normal embryonic development of the PV. Usually, the PV develops from week 5 to 10 of gestational life by selective involution of the peri-intestinal vitelline venous loop, while the IVC is formed from the combination of a common hepatic vein with various embryologic venous channels, which helps in the development of the hepatic segment of the IVC. Exaggerated involution between weeks 4 and 10 of gestational life can lead to the total absence of the PV, ultimately resulting in AM with abnormal mesentericcaval shunt [6].

Type I AM is usually observed in younger individuals with female predominance and is generally accompanied by abnormalities involving the heart, liver, gastrointestinal tract, and blood vessels, while Type II is rarer, frequently observed in adults, has male predominance, and is usually not associated with other abnormalities [7]. Patients with AM have a wide variety of symptoms ranging from those asymptomatic patients found incidentally in adulthood to those with congenital severe hepatic manifestations identified at birth. Usually, the diagnosis is reached through Doppler ultrasound. Moreover, the magnetic resonance angiography and computed tomography angiography are used for further classification of shunt and assessment of associated anomalies [8].

Likewise, our patient was a 24-year-old male who was asymptomatic till now, developed insidious-onset dyspnea and fever that compelled him to seek medical attention. CTPA and CECT chest demonstrated findings of Type $1 \mathrm{PH}$. Thus, Tadalafil-Bosentan was initiated. However, the dyspnea worsened with the presence of hemoptysis, hypotension, and raised CVP. On further evaluation, raised arterial ammonia caught our attention, and CECT of abdomen and pelvis led us to the diagnosis of Type II AM. It is reported that despite the presence 
of an uninterrupted flow of blood from portal to systemic venous systems, ammonia levels are raised in only $26 \%$ of patients with AM, and only $15 \%$ of all them experience HE [9]. Though our patient had raised ammonia levels, there was no clinical evidence of HE.

Around 2\% of patients with PHT develop PH resulting in a condition collectively termed as portopulmonary hypertension (PPHT) [10]. Among various complications of AM, PPHT significantly affects the survival and requires long-term treatment [11]. The pathogenesis of PPHT in patients with AM can be explained by several factors, including an asymmetry between the constrictors and dilators of the vessels, thereby resulting in pulmonary vasoconstriction; the state of hyperdynamic circulation favors the development of $\mathrm{PH}$; the raised pulmonary circulation may contribute to extra shear stress that results in injury to the endothelial tissue, and dysfunction with vasoconstriction and dynamic vascular remodeling; and the proliferative and vasoconstrictive molecules that arrive in the lungs, without being detoxified in the liver, act on the pulmonary vessels and may cause $\mathrm{PH}$ [12]. Absence of $\mathrm{HE}$ in our patient is worth highlighting, as very few patients are diagnosed in isolation.

Treatment of AM depends on the variant of shunt identified. Patients with Type I AM require prolonged follow-up with clinical, laboratory, and radiological examinations and LT being definitive treatment, while those with Type II AM need shunt closure at the earliest. In the majority of the patients, shunt closure leads to restoration of intrahepatic portal blood flow. Moreover, symptomatic improvement and stabilization of various complications (pulmonary, neurological, cardiac, and renal) are reported. Closure of the shunt can be performed either surgically or percutaneously [13]. Zhou et al. used surgical ligation for patients with Type II AM having giant shunt [14]. Our center has the facilities of interventional radiology. In our patient, the shunt was large and closure with a stent was not possible. Thus, ASD graft was used and successful closure was achieved.

The available literature reports recurrence and development of further complications among patients with Type II AM. Li et al. reported recurrence of hyperammonemia after 3 months of shunt closure in a patient with Type II AM [9]. Iida et al. reported a case of Type II AM that was managed with surgical ligation of shunt. However, after 4-years, the patient had a recurrence of $\mathrm{PH}$ due to the development of intrahepatic portosystemic shunts that were treated with LT [15]. Though after 3 months, our patient was free from any complaints, but he is being followed up further to look for the development of any further complications.

\section{Conclusion}

Our case demonstrates a rare, but reversible cause of $\mathrm{PH}$. Though extremely rare, Type II AM should be included in the differential diagnosis of a patient presenting with dyspnea and provisionally diagnosed as a case of $\mathrm{PH}$. Once diagnosed, the shunt closure should be performed immediately to prevent the development of further complications. Upon shunt closure, the patients should be followed on a long-term basis to identify any complications or recurrence.

\begin{abstract}
Abbreviations
AM: Abernethy malformation; ANA: Antinuclear antibody; ANCA: Antineutrophil cytoplasmic antibody; CECT: Contrast-enhanced computed tomography; CTPA: Computed tomography pulmonary angiogram; CVP: Central venous pressure; HE: Hepatic encephalopathy; Hg: Mercury; IVC: Inferior vena cava; LT: Liver transplantation; PASP: Pulmonary artery systolic pressure; PV: Portal vein; PH: Pulmonary hypertension; PHT: Portal hypertension; PPHT: Porto-pulmonary hypertension
\end{abstract}

\section{Acknowledgements}

The authors would like to thank Dr. Vikas S. Sharma (MD), Principal Consultant, Maverick Medicorum ${ }^{\text {TM }}$ (India), for medical writing assistance in the preparation of this article.

\section{Authors' contributions}

AA analyzed and interpreted the patient data and revised the final manuscript. DB analyzed and interpreted the patient data and supervised the patient during hospital admission. SP participated in the interpretation of the interventional data of the patient. VMS was a major contributor in writing the manuscript. All authors read and approved the final manuscript.

\section{Funding}

The authors received no funding for this study.

Availability of data and materials

Not applicable.

\section{Declarations}

Ethics approval and consent to participate Not applicable.

Consent for publication

Oral and written informed consents were obtained from the patient or from his eligible relatives.

\section{Competing interests}

The authors declare that they have no competing interests.

Received: 3 July 2021 Accepted: 17 August 2021

Published online: 23 November 2021

References

1. Kumar A, Kumar J, Aggarwal R, Srivastava S (2008) Abernethy malformation with portal vein aneurysm. Diagn Interv Radiol 14(3):143-146

2. Peček J, Fister P, Homan M (2020) Abernethy syndrome in Slovenian children: five case reports and review of literature. World J Gastroenterol 26(37):5731-5744. https://doi.org/10.3748/wjg.v26.137.5731

3. Abernethy J (1797) Account of two instances of uncommon formation in the viscera of the human body: from the philosophical transactions of the Royal Society of London. Med Facts Obs 7:100-108

4. Morgan G, Superina R (1994) Congenital absence of the portal vein: two cases and a proposed classification system for portasystemic vascular anomalies. J Pediatr Surg 29(9):1239-1241. https://doi.org/10.1016/0022-34 68(94)90812-5 
5. Lin Y, Li X, Li S, Ba H, Wang H, Zhu L (2020) Treatment option for Abernethy malformation-two cases report and review of the literature. Front Pediatr 8: 497447. https://doi.org/10.3389/fped.2020.497447

6. Marks C (1969) Developmental basis of the portal venous system. Am J Surg 117(5):671-681. https://doi.org/10.1016/0002-9610(69)90404-8

7. Stepffer C, Marques A, Barbosa JD, Ferrario S, Haag D (2020) Pulmonary arterial hypertension in a patient with a Portosystemic shunt: diagnostic challenge. Case (Phila) 4(2):93-96. https://doi.org/10.1016/j.case.2019.08.007

8. Calderón MPM, Robles ACR, Astudillo MEL, Benenaula JFF, Tenecela MJT (2019) Malformation of Abernethy type 2: presentation of a clinical case and review of literature. Pharm Pharmacol Int J 7(5):209-212. https://doi.org/10.1 5406/ppij.2019.07.00253

9. Li H, Ma Z, Xie Y, Tian F (2017) Recurrent Hyperammonemia after Abernethy malformation type 2 closure: a case report. Ann Hepatol 16(3):460-464. https://doi.org/10.5604/16652681.1235492

10. Hervé $P$, Lebrec $D$, Brenot F, Simonneau G, Humbert M, Sitbon O, Duroux $P$ (1998) Pulmonary vascular disorders in portal hypertension. Eur Respir J 11(5):1153-1166. https://doi.org/10.1183/09031936.98.11051153

11. Zhang XL, Duan XM, Wang FY, Zhang X, Sun Y, Ma N, Du ZD (2017) An infant with Abernethy malformation associated with Heterotaxy and pulmonary hypertension. Chin Med J 130(18):2257-2258. https://doi.org/1 0.4103/0366-6999.213978

12. Raevens S, Geerts A, Van Steenkiste C, Verhelst X, Van Vlierberghe H, Colle I (2015) Hepatopulmonary syndrome and portopulmonary hypertension: recent knowledge in pathogenesis and overview of clinical assessment. Liver Int 35(6):1646-1660. https://doi.org/10.1111/liv.12791

13. Sahu MK, Bisoi AK, Chander NC, Agarwala S, Chauhan S (2015) Abernethy syndrome, a rare cause of hypoxemia: a case report. Ann Pediatr Cardiol 8(1):64-66. https://doi.org/10.4103/0974-2069.149526

14. Zhou M, Zhang J, Luo L, Wang B, Zheng R, Li L, Jing H, Zhang S (2020) Surgical ligation for the treatment of an unusual presentation of type II Abernethy malformation. Ann Vasc Surg 65:285.e1-285.e5. https://doi.org/1 0.1016/j.avsg.2019.10.094

15. lida T, Ogura Y, Doi H, Yagi S, Kanazawa H, Imai H, Sakamoto S, Okamoto S, Uemoto $S$ (2010) Successful treatment of pulmonary hypertension secondary to congenital extrahepatic portocaval shunts (Abernethy type 2) by living donor liver transplantation after surgical shunt ligation. Transpl Int 23(1):105-109. https://doi.org/10.1111/j.1432-2277.2009.00964.x

\section{Publisher's Note}

Springer Nature remains neutral with regard to jurisdictional claims in published maps and institutional affiliations.

\section{Submit your manuscript to a SpringerOpen ${ }^{\circ}$ journal and benefit from:}

- Convenient online submission

- Rigorous peer review

- Open access: articles freely available online

- High visibility within the field

- Retaining the copyright to your article

Submit your next manuscript at $\boldsymbol{\nabla}$ springeropen.com 